Cahiers Société

\title{
Marx et la critique de la technique : réflexions à partir des Grundrisse et du Capital
}

\section{Maxime Ouellet}

Numéro 2, décembre 2020

Marx, critique du capital et de la société

URI : https://id.erudit.org/iderudit/1075552ar

DOI : https://doi.org/10.7202/1075552ar

Aller au sommaire du numéro

\section{Éditeur(s)}

Collectif Société

ISSN

2562-5373 (imprimé)

2562-5381 (numérique)

Découvrir la revue

Citer cet article

Ouellet, M. (2020). Marx et la critique de la technique : réflexions à partir des Grundrisse et du Capital. Cahiers Société, (2), 23-43.

https://doi.org/10.7202/1075552ar
Résumé de l'article

Cet article consiste en une réflexion sur la nature de la technique dans les écrits de Marx. Dans un premier temps, la manière dont Marx aborde la question de la technique à partir de son ontologie de l'autoproduction de l'être générique sera présentée. Par la suite, la critique marxienne du machinisme sera analysée à l'aune de son ontologie de l'autoproduction. L'analyse du machinisme proposée par Marx dans le chapitre XIII du Capital permettra de faire un retour critique sur le désormais célèbre passage des Grundrisse communément désigné en tant que « Fragment sur les machines », et qui est mobilisé par de nombreux théoriciens contemporains du post-capitalisme tels que les cognitivistes ou les accélérationnistes. Contre leur vision productiviste, il s'agira de proposer une lecture qui permettrait de dépasser les apories auxquelles mènent les analyses unilatérales du développement technologique sous le capitalisme, que celles-ci soient progressistes ou romantiques. 


\title{
Marx et la critique de la technique : réflexions à partir des Grundrisse et du Capital
}

\author{
Maxime OUELLET \\ Université du Québec à Montréal
}

Cet article vise à proposer une réflexion sur la nature de la technique dans les écrits de Marx. Nous poserons la question visant à savoir si Marx peut être considéré comme un « penseur de la technique » pour reprendre le titre de l'ouvrage de Kostas Axelos ${ }^{1}$ paru en 1961. Cette question apparaît d'autant plus pertinente que l'enjeu de la technique semble un point aveugle de la plupart des écrits marxistes. En effet selon la vulgate, avec l'augmentation des forces productives, le progrès économique et social serait de plus entravé par le marché et la propriété privée. Dans ce contexte, le développement technologique mènerait automatiquement vers une société post-capitaliste à condition de changer les rapports de propriété. Ce type d'analyse repose sur une théorie a-critique postulant à la fois la neutralité de la technique et une certaine forme de déterminisme $^{2}$. Selon le marxisme traditionnel, le progrès technologique se développerait de manière autonome et ce serait le bon ou le mauvais usage qu'on en fait qui serait la cause de ses effets néfastes ou émancipateurs. Cette conception de la technique est condensée dans la pensée léniniste dont on peut encore ressentir l'influence chez certains penseurs marxistes contemporains. On retrouve de tels postulats chez les théoriciens du capitalisme cognitif comme Hardt et Negri, ou encore de manière encore plus caricaturale chez les théoriciens de l'accélérationnisme comme Srnicek et Williams, qui s'appuient d'ailleurs explicitement sur Lénine dans leur Manifeste accélérationniste :

En réalité, comme Lénine l'a écrit en 1918 dans son texte «Sur l'infantilisme "de gauche" " : « Le socialisme est impossible sans la technique du grand capitalisme, conçue d'après le dernier mot de la science la plus moderne, sans une organisation d'État méthodique qui ordonne des dizaines de millions d'hommes à l'observation la plus rigoureuse d'une norme unique dans la production et la

\footnotetext{
${ }^{1}$ Kostas Axelos, Marx penseur de la technique, Paris, Minuit, 1961.

2 Pour une typologie des différentes théories de la technique dans la pensée sociale, voir Andrew Feenberg, (Re) penser la technique. Vers une technologie démocratique, Paris, La Découverte, 2004.
} 
répartition des produits. Nous, les marxistes, nous l'avons toujours affirmé ; quant aux gens qui ont été incapables de comprendre au moins cela (les anarchistes et une bonne moitié des socialistesrévolutionnaires de gauche), il est inutile de perdre même deux secondes à discuter avec eux ${ }^{3} \gg$.

On retrouve à l'opposé dans certaines relectures contemporaines de Marx une critique de la domination technologique au sein du capitalisme s'appuyant sur la pensée d'Heidegger. Initiée par Kostas Axelos dans les années 19604, « le branchement d'Heidegger sur Marx 5 » trouve sa forme la plus achevée à l'heure actuelle dans l'œuvre de Jean Vioulac 6 . En dépit de sa portée critique indéniable, l'analyse heideggériano-marxienne de la domination planétaire du dispositif technique capitaliste n'en demeure pas moins problématique. Bien qu'elle s'oppose avec raison à la conception de la neutralité de la technique du marxisme traditionnel, la perspective essentialiste défendue par le marxisme heideggérien néglige la spécificité sociohistorique du capitalisme, ce qui est pourtant l'objet même de la critique marxienne de ce mode de production. L'analyse heideggérienne repose en effet sur une critique unilatérale de la modernité. Combinée à la vision téléologique de la philosophie hégélienne de l'histoire, cette lecture conduit à la conclusion que la société capitaliste - qui repose sur une technique conçue comme le fondement transhistorique du rapport instrumental au monde - est le parachèvement de la métaphysique occidentale telle que théorisée depuis Platon à l'époque de l'Antiquité grecque. Or, ce type de lecture ne permet pas d'expliquer pourquoi la logique métaphysique de quantification du monde n'est jamais apparue comme imaginaire structurant la pratique sociale dans l'Antiquité, ni pourquoi il a fallu plus de vingt siècles pour que se réalise enfin le projet de domination planétaire de la raison calculatrice ${ }^{8}$. La relecture heideggérienne de Marx nous oblige à abandonner tout espoir dans la possibilité de la théorie critique à

\footnotetext{
3 Nick Srnicek et Alex Williams, «Manifeste accélérationniste », Multitudes, n 56, 2014 ; en ligne : http://www.multitudes.net/manifeste-accelerationniste/.

4 Kostas Axelos, Marx penseur de la technique, op. cit.

5 La formule est du philosophe français Gérard Granel qui propose également une interprétation heideggérienne de Marx. Pour une présentation de sa pensée, voir Richard Sobel, «Production et infini : l'hypothèse Marx-Granel sur l'origine du capitalisme », Economia, vol. 7, nº 4, 2017, p. 513-544.

6 Voir Jean Vioulac, L'époque de la technique. Marx, Heidegger et l'accomplissement de la métaphysique, Paris, Presses Universitaires de France, 2009; La logique totalitaire. Essai sur la crise de l'Occident, Paris, Presses Universitaires de France, 2013; Apocalypse de la vérité: Méditations heideggériennes, Paris, Ad Solem, 2014 ; Science et révolution. Recherche sur Marx, Husserl et la phénoménologie, Paris, Presses Universitaires de France, 2015 ; Approche de la criticité : Philosophie, capitalisme, technologie, Presses Universitaires de France, 2018.

7 Le concept heideggérien de dispositif réfère à l'essence de la technique moderne qui en augmentant l'efficacité sépare l'humain du contexte dans lequel sont produits les biens. La technique moderne induit ainsi une séparation entre les moyens et les fins qui produit un processus d'arraisonnement du monde, c'est-à-dire de transformation de la nature et de l'humain en ressources à exploiter.

8 Cornelius Castoriadis, La Cité et les Lois. Ce qui fait la Grèce. Séminaires 1983-1984. La création humaine III, Paris, Seuil, 2008.
} 
éclairer le présent en vue de penser un autre monde et à accepter la déclaration de Heidegger selon laquelle « Seul un Dieu peut encore nous sauver9 ».

Contre le diagnostic pour le moins unilatéral proposé par les relectures heideggériennes de Marx, il s'agira de montrer qu'il existe chez l'auteur du Capital certains éléments conceptuels permettant d'élaborer une théorie critique et dialectique de la technique. L'attaque unilatérale contre la raison et la technique abstraite repose sur une analyse non dialectique qui conduit souvent à une vision dualiste où la pensée de Marx oscille entre un romantisme qui déplore la déshumanisation du processus d'industrialisation et un positivisme qui l'admire. Vioulac résume bien les postulats du marxisme traditionnel concernant la technique :

tout le propos de Marx consiste à différencier technique et capitalisme : c'est-à-dire à mettre en évidence que cette Machinerie tourne avec le logiciel capitaliste, mais à postuler qu'elle pourrait tout aussi bien tourner avec le logiciel communiste, et que la contradiction interne à sa logique de fonctionnement fonde la possibilité du passage d'un logiciel à l'autre - et c'est pourquoi il n'y a pas chez Marx de critique de la technique en tant que telle ${ }^{10}$.

Pour sortir de cette impasse, il apparaît essentiel de retourner à l'une des inspirations fondamentales de la pensée marxienne, c'est-à-dire Aristote ${ }^{11}$. La prise en considération de l'influence d'Aristote dans la pensée de Marx est essentielle puisqu'elle permet de mieux saisir le «renversement» qu'il tente d'opérer par rapport au système hégélien ${ }^{12}$. Elle permet également de dénouer un certain nombre d'apories auxquelles

\footnotetext{
9 Entretien de Martin Heidegger dans Der Spiegel tenu le 23 septembre 1966, publié le 31 mai 1976 ; en ligne : http://www.accordphilo.com/2018/01/seul-un-dieu-peut-encore-nous-sauver.html.

10 Jean Vioulac, La logique totalitaire, op. cit., p. 453.

${ }^{11}$ Nous reprenons ici la thèse de Pierre Rodrigo qui identifie trois sources d'inspiration philosophiques dans l'ontologie marxienne de l'autoproduction, Hegel, Feuerbach et Aristote. Pierre Rodrigo, Sur l'ontologie de Marx. Auto-production, travail aliéné et capital, Paris, Vrin, 2014.

12 Selon Annick Jaulin, « le rapport Hegel/Marx s'inscrit dans un schème analogue à celui du débat entre Platon et Aristote sur la nature et le statut des Idées" ("Marx Lecteur d'Aristote », Les études philosophiques, vol. 1, $\mathrm{n}^{\circ} 161,2016$, p. 105-122). On pourrait même affirmer suivant Castoriadis que si Marx est l'Aristote des temps modernes, c'est parce qu'il essaie de faire jouer le rôle de Platon à Hegel. En fait, c'est plutôt à Kant que devrait revenir le personnage du Platon des temps modernes. C'est du moins l'hypothèse qui sous-tend cet article, s'inspirant notamment de l'excellente présentation par Marcuse de la pensée de Hegel et de sa continuité dans l'œuvre de Marx. Voir Herbert Marcuse, Raison et Révolution, Paris, Minuit, 1968. Il ne s'agit pas ici de nier l'influence de la dialectique hégélienne dans la pensée de Marx, ce qui est courant et très problématique dans les lectures contemporaines de Marx, et plus spécifiquement chez les auteurs français d'inspiration spinoziste ou heideggérienne, mais plutôt de spécifier les similitudes et les discontinuités de leurs pensées respectives. En fait, il est possible d'interpréter l'œuvre de Marx comme une tentative jamais totalement aboutie de dépassement de la dialectique hégélienne. Comme le souligne à juste titre Jacques D'Hondt : " le marxisme n'est pas l'hégélianisme. Mais Marx et Engels n'ont pas renié Hegel. Ils ne l'ont pas traité comme un "chien crevé", à la manière des critiques grincheux et incompréhensifs qui s'en prenaient à eux en même temps qu'à lui. Ils ont
} 
conduit sa théorie critique de la modernité capitaliste. Aporie qui se décline ainsi : comment maintenir et même épanouir encore les valeurs de liberté, d'égalité et d'individualité qui sont corollaires de la modernité capitaliste, et principalement de son dispositif technoscientifique, tout en supprimant la médiation aliénante qui l'a rendue possible ${ }^{13}$ ? Bref, est-il possible de penser un autre rapport à la technique qui ne soit pas capitaliste ? À ce titre, il sera soutenu que c'est principalement à partir de son analyse de la nature illimitée de la logique de l'accumulation du capital qu'il est possible d'entrevoir chez Marx une critique de la technique dans le capitalisme qui permette de dépasser les apories qu'on retrouve au sein des lectures soit essentialistes ou déterministes de sa pensée.

Ce texte comporte trois parties. Dans un premier temps, la manière dont Marx aborde la question de la technique à partir de son ontologie de l'autoproduction de l'être générique sera présentée. Par la suite, la critique marxienne du machinisme sera évaluée à l'aune de son ontologie de l'autoproduction. L'analyse du machinisme proposée par Marx dans le chapitre XIII du Capital permettra de faire un retour critique sur le désormais célèbre passage des Grundrisse intitulé « Fragment sur les machines » qui est mobilisé par de nombreux théoriciens contemporains du post-capitalisme tels que les cognitivistes ou les accélérationnistes ${ }^{14}$. Contre leur vision productiviste, il s'agira de proposer une lecture qui permettrait de dépasser les apories auxquelles mènent les analyses unilatérales du développement technologique sous le capitalisme, que celles-ci soient progressistes ou romantiques.

\section{Marx et la question de la technique : premier abord}

Comme le soutient le philosophe Pierre Rodrigo, à la question de savoir si Marx est un penseur de la Technique - Technique avec un $T$ majuscule - au sens d'une abstraction idéalisante comme l'entend Heidegger, il faut répondre par la négative ${ }^{15}$. Son rapport à la question de la technique doit être compris au sein de son analyse de la genèse historique du mode de production capitaliste. Il convient de rappeler que pour Marx les catégories d'analyse économique comme celles de travail, de marchandise, de valeur, de capital, de même que les catégories de la technique, comme celles d'instrument de travail, de force productive ou de machine, n'ont un sens que pour une époque historique particulière, celle de la modernité capitaliste.

respecté comme un maître, dont ils avaient beaucoup appris » (Jacques D’Hondt, De Hegel à Marx, Paris, Presses Universitaires de France, 1972, p. 209).

13 Jean-Joseph Goux, La Frivolité de la valeur. Essai sur l'imaginaire du capitalisme, Paris, Blusson, 2000.

14 Karl Marx, Fondements de la critique de l'économie politique, vol. 2, Chapitre du Capital, trad. R. Dangeville, Paris, Anthropos, 1968 (1858).

15 Pierre Rodrigo, « Marx penseur et la technique », Philosophie, vol. 2, nº 133, 2017, p. 37-51. 
En ce sens, si aux yeux de Marx un fétichisme se trouve au cœur de la pensée économique en ce qu'elle présente ses catégories comme étant naturelles et transhistoriques, ce même fétichisme opère lorsque la pensée économique traditionnelle traite de la technique comme un outil neutre.

Il faut également se garder de comprendre Marx comme un penseur du déterminisme technique telle qu'une lecture unilatérale de son œuvre pourrait le laisser entendre. Par exemple, plusieurs ont vu dans un passage de Misère de la philosophie, une forme de déterminisme technique :

Les rapports sociaux sont intimement liés aux forces productives. En acquérant de nouvelles forces productives, les hommes changent leur mode de production, et en changeant le mode de production, la manière de gagner leur vie, ils changent tous leurs rapports sociaux. Le moulin à bras vous donnera la société avec le suzerain ; le moulin à vapeur, la société avec le capitaliste industriel16.

Contre cette lecture non dialectique, il faut plutôt voir en Marx un analyste des conditions sociales et historiques de possibilité du capitalisme. Cela signifie que Marx s'oppose à l'idéologie moderne de la neutralité de la technique, sa configuration structure déjà a priori l'horizon des possibles sans présupposer qu'elle les détermine directement. Le mode de production capitaliste a également comme condition de possibilité, outre un certain dispositif technique, un ensemble de médiations culturelles, idéologiques et politiques. La catégorie de la valeur, par exemple, ne peut surgir que dans une société fondée sur un imaginaire égalitariste. Marx dira à ce sujet que : « [1]e secret de l'expression de la valeur, l'égalité et l'égale validité de tous les travaux parce que et pour autant que ceux-ci sont du travail humain en général, ne peut être déchiffré qu'à partir du moment où le concept d'égalité humaine a acquis la solidité d'un préjugé populaire ${ }^{17} \gg$. La condition de possibilité du mode de production capitaliste est intrinsèquement politique, cette nouvelle manière de structurer les rapports économiques est impensable sans la mise en place de la révolution bourgeoise. L'échange marchand ne peut être possible qu'à partir de la réduction de l'individu à son plus petit dénominateur commun, celui de force de travail, qui résulte d'un procès historique qui a dissout les institutions corporatives qui protégeaient l'individu des aléas du marché. Il ne faut cependant pas confondre le bourgeois et le capitaliste ${ }^{18}$. Si la révolution bourgeoise est une condition nécessaire à l'apparition du capitalisme, elle n'est pas suffisante. Si l'on suit l'analyse de Marx telle qu'exposée dans le chapitre XXIV du Capital portant sur l'accumulation primitive, c'est au sein des campagnes anglaises qu'il faut trouver

\footnotetext{
${ }^{16}$ Karl Marx, Misère de la philosophie. Réponse à la philosophie de la misère de Proudhon, 1847 ; en ligne : https://www.marxists.org/francais/marx/works/1847/06/km18470615.htm.

17 Karl Marx, Le Capital, livre 1 : Le procès de production du capital, trad. J.-P. Lefebvre, Paris, Presses Universitaires de France, 1993, p. 68.

18 Ellen Meiksins Woods, L'origine du capitalisme. Une étude approfondie, Montréal, Lux, 2009.
} 
l'origine du capitalisme. C'est le processus d'enclosure des terres qui a rendu possible la révolution capitaliste. Selon Ellen Meiksins Woods, seule l'Angleterre avait mis en place des impératifs structurels à l'accroissement illimité de la productivité au moyen du développement technologique à la suite de la séparation des paysans de leurs moyens de subsistance. La bourgeoisie française, par exemple, n'avait pas réuni ces conditions. Ce n'est qu'à la suite des pressions extérieures induites par la compétition internationale que la France et les autres pays occidentaux ont été contraints d'intégrer les impératifs liés à l'accroissement illimité des forces productives.

L'analyse de Marx ne se limite toutefois pas en une genèse des conditions historiques de possibilité du capitalisme ; elle a également la prétention de fournir une théorie critique du capitalisme et du rapport au monde instrumental qu'il institue. Il est essentiel de comprendre que la critique marxienne du rôle de la technique dans le capitalisme s'appuie aussi sur une position ontologique, qu'on peut nommer une ontologie de la production, ou plutôt de l'autoproduction'19, qu'il élabore à partir de ses écrits de jeunesse portant notamment sur le concept d'aliénation. La catégorie de l'aliénation traverse l'entièreté de l'œuvre de Marx, contrairement à ce que prétend l'analyse althussérienne qui soutient qu'elle implique une anthropologie naturaliste qu'il aurait abandonnée dans ses écrits de maturité. C'est à partir d'une conception ontologique de la subjectivité objective²o telle que définie dans les Manuscrits de 1844 - où il réfère à l'être humain comme un sujet social et historique - que Marx est en mesure de critiquer dans Le Capital l'aliénation induite par le dispositif capitaliste.

C'est pourquoi il est nécessaire à ce stade de l'exposé de rappeler la dette que Marx a contractée envers Aristote dans la formulation de son ontologie de l'autoproduction de l'être générique. En fait, la lecture d'Aristote par Marx remonte à sa thèse de doctorat portant sur La différence de la philosophie naturelle chez Démocrite et chez Épicure (1841). Marx voit en Aristote le penseur à partir duquel il est possible de sortir du système spéculatif hégélien. C'est au moyen d'une reprise de la critique aristotélicienne de Platon portant sur la question du rapport entre le sujet et le prédicat que Marx tente d'opérer le dépassement de l'opposition entre l'idéalisme de Hegel et le matérialisme de Feurbach. Marx voit donc chez Aristote, plutôt que chez Hegel, le penseur à partir duquel il est possible de réconcilier l'idéalité de la pensée et la réalité objective $^{21}$. Aristote explique dans son traité sur l'âme ${ }^{22}$ que dans la pensée platonicienne les prédicats abstraits séparés des sujets deviennent eux-mêmes des sujets que l'on dote d'une existence autonome. Aristote reproche à Platon de faire coexister

\footnotetext{
19 Pierre Rodrigo, Sur l'ontologie de Marx, op. cit.

${ }^{20}$ On pourrait également utiliser la notion d'ontologie de l'être social à la manière de Lukács.

${ }^{21}$ Pierre Rodrigo, Sur l'ontologie de Marx, op. cit., p. 111.

22 Aristote, De l'âme, Paris, Flammarion, 1993.
} 
deux mondes, un monde réel et un monde idéel, les idéalités acquérant ainsi une existence substantielle ${ }^{23}$.

Marx reprend à son compte la critique aristotélicienne des idées platoniciennes, c'est-à-dire l'inversion du sujet et du prédicat, afin de fonder son ontologie de l'autoproduction. L'ontologie marxienne de l'autoproduction repose sur la méthode de déduction sociale des catégories de la pensée qui vise à saisir les conditions sociales et historiques de possibilité de toute forme de connaissance. En ce sens, sera considérée par Marx comme « idéologique » toute forme de connaissance qui ne reconnaît pas son fondement historique dans la pratique sociale.

Ceci ne signifie donc pas que les idées ne sont qu'un voile masquant la réalité des rapports de classe comme le soutient le marxisme vulgaire. La notion de fétichisme de la marchandise chez Marx vise au contraire à montrer que le capital consiste en une abstraction réelle, c'est-à-dire que son mode opératoire repose sur une inversion entre l'idée abstraite et la pratique concrète ; bref, c'est la forme sociale qui donne son contenu à la pratique matérielle. C'est pourquoi il ne faut pas opposer selon Marx la valeur d'échange à la valeur d'usage comme le font les économistes, puisqu'elles sont toutes les deux médiatisées par la forme-valeur. C'est également à partir de la distinction qu'Aristote fait entre chrématistique et oikonomia que Marx construira son analyse de la forme-valeur. Si pour Aristote l'échange économique qualifié de naturel est médiatisé par des normes sociales qui instituent des limites à son expansion, la chrématistique repose au contraire sur une forme pervertie de l'échange qui se caractérise par son caractère illimité. La chrématistique est jugée par Aristote comme une forme pervertie de l'échange puisqu'elle possède un caractère potentiellement destructeur pour les normes de la vie bonne instituées dans la cité. C'est sur cette base que Marx construit sa critique du fétichisme de la marchandise. Selon Marx, la valeur consiste en une pure idéalité qui se transforme en sujet réel. Le fétichisme consiste donc à inverser le rapport entre sujet mystique et sujet concret ${ }^{24}$. Ainsi dira-t-il dans le chapitre I du Capital que :

Dans ce monde-là, les produits du cerveau humain semblent être figures autonomes, douées d'une vie propre, entretenant des rapports les unes avec les autres et avec les humains. Ainsi en va-t-il dans le monde marchand des produits de la main humaine. J'appelle cela le fétichisme, fétichisme qui adhère aux produits du travail dès lors qu'ils sont produits comme marchandises, et qui, partant, est inséparable de la production marchande25.

\footnotetext{
23 Annick Jaulin, op. cit., p. 110.

24 Nous verrons plus bas que la catégorie du fétichisme ne s'applique pas uniquement à la marchandise, mais également au dispositif de production technique qui, dans le capitalisme, acquiert une dimension fétichisée.

25 Karl Marx, Le Capital, livre 1, op. cit., p. 83 ; nous soulignons.
} 
Tout porte à croire que cette analogie entre la main et le cerveau, Marx la tire également d'Aristote qui, dans un passage des Parties des Animaux, conteste la thèse d'Anaxagore pour qui «l'homme est le plus intelligent des animaux parce qu'il a des mains ${ }^{26} »$. Pour Aristote, ce rapport est inversé ; l'intelligence et la possession des mains sont une spécificité de l'animal humain, peu importe le sens dans lequel on établit leur relation ${ }^{27}$. C'est ce qui distingue pour Marx l'être organique de l'être générique :

Par la production pratique d'un monde objectif, le façonnement de la nature non organique, l'homme s'affirme comme être générique conscient, c'est-à-dire comme un être qui se comporte à l'égard de l'espère humaine comme il se comporte à l'égard de sa propre essence, ou à l'égard de soi en tant qu'être générique. Certes l'animal aussi produit. Il se construit un nid, des habitations, comme l'abeille, le castor, la fourmi, etc. Mais il ne produit que ce dont il a immédiatement besoin pour lui et son petit; il produit d'une façon unilatérale, tandis que l'homme produit d'une façon universelle; il ne produit que sous l'emprise du besoin physique immédiat, tandis que l'homme produit même lorsqu'il est libéré de tout besoin physique et ne produit vraiment que lorsqu'il est vraiment libéré [...]. L'animal ne façonne que selon la mesure et selon les besoins de l'espèce à laquelle il appartient, tandis que l'homme sait produire à la mesure de toute espèce et sais appliquer partout à l'objet sa nature inhérente. C'est pourquoi l'homme façonne aussi d'après les lois de la beauté. C'est précisément en façonnant le monde des objets que l'homme s'affirme réellement comme un être générique. Cette production est sa vie générique active. Grâce à cette production, la nature apparaît comme son œuvre et sa réalité. L'objet du travail est donc l'objectivation de la vie générique de l'homme, car il ne se dédouble pas lui-même de façon seulement intellectuelle, comme c'est le cas dans la conscience, mais activement, réellement, et il se contemple luimême dans un monde qu'il a lui-même créé28.

Marx reprendra cette même analogie animalière dans le chapitre $\mathrm{V}$ du Capital portant sur la distinction entre le procès de travail et le procès de valorisation lorsqu'il soutient que :

Une araignée accomplit des opérations qui s'apparentent à celles du tisserand, et une abeille en remontre à maint architecte humain dans la construction de ses cellules. Mais ce qui distingue d'emblée

\footnotetext{
${ }^{26}$ Annick Jaulin, op. cit., p. 121.

27 Idem.

28 Karl Marx, Manuscrits de 1844, Paris, Flammarion, 1996, p. 114.
} 
le plus mauvais architecte de la meilleure abeille, c'est qu'il a construit la cellule dans sa tête avant de la construire dans la cire29.

La distinction entre l'humain et l'animal repose sur le fait qu'alors que l'animal se rapporte immédiatement à la nature, l'humain médiatise son rapport avec celle-ci au moyen de concepts qui lui permettent de s'élever vers son genre, c'est-à-dire 1'universel humain. L'autoproduction de l'être générique pour Marx présuppose donc une conception de l'être humain qu'il définit comme un "toolmaking animalso », c'est-àdire qu'il s'agit d'un être qui produit son monde grâce au maniement des outils. Il faut ici se garder de voir dans la conception marxienne de l'aliénation une anthropologie naturaliste et transhistorique comme l'ont fait la plupart des marxistes à la suite d'Althusser. La conception marxienne de l'aliénation correspond à la dépossession de la nature sociale de l'être humain, qui ne doit pas être comprise au sens transhistorique, mais telle qu'elle s'est constituée historiquement. Avant d'être aliénation d'un savoirfaire, le capitalisme est d'abord l'aliénation d'un savoir-être constitué historiquement et objectivé dans la culture. En effet, Marx ne dit pas que l'être humain est par nature un fabricant d'instruments, il s'agit là selon lui d'un préjugé qu'il attribue à la mentalité américaine. Marx soutient, contrairement à Aristote cette fois-ci, que si l'homme n'est pas par nature un animal politique, il est naturellement un animal social. Dans une note de bas de page du Capital il ajoutera : " La définition d'Aristote est que l'homme est par nature citoyen. Elle est aussi caractéristique de l'Antiquité classique que l'est la mentalité yankee selon laquelle l'homme est par nature fabricant d'instruments ${ }^{31}$ ».

Il reste que si Marx définit l'être humain comme un être social, et que dans la société capitaliste apparait la forme subjective du «toolmaking animal», l'aliénation du travail comporte nécessairement une dimension technique qui correspond en une aliénation d'un savoir-faire32. La conception de la technique déployée par Marx lorsqu'il traite du procès de travail général - c'est-à-dire en faisant abstraction du procès de valorisation capitaliste - rejoint le sens grec de technè tel que défini par Aristote : « La technè en général ou bien imite la physis ou bien effectue ce que la nature est dans l'impossibilité d'accomplir33 ». C'est au moyen de l'outil, qui est une extension de sa main et de son savoir-faire, donc de son intelligence, que l'être humain peut avoir une mainmise sur le monde qu'il a créé. L'usage de la technique dans les sociétés antérieures au capitalisme demeure enchâssé dans un ensemble de médiations qui viennent conférer une finalité sociale à la pratique. L'outil médiatise le rapport de l'humain à la nature afin de satisfaire des besoins qui sont socialement constitués, ce qui implique le caractère fini d'une production de richesse matérielle qui va s'opposer à l'infinitude

\footnotetext{
29 Karl Marx, Le Capital, livre 1, op. cit., p. 200.

30 Ibid., p. 202.

31 Ibid., p. 367.

32 Kostas Axelos, Marx penseur de la technique, op. cit.

33 Cité par Cornelius Castoriadis, "Technique », dans Les carrefours du labyrinthe, I, Paris, Seuil, 1968 , p. 301
} 
de la production de la richesse abstraite qu'est la valeur. C'est au moment de la séparation entre la pratique et la connaissance de celle-ci, de l'aliénation d'un savoir-faire, que s'enclenche le processus d'aliénation spécifiquement capitaliste. Le travailleur aliéné est celui qui a été dépossédé des conditions objectives de réalisation de son genre comme être universel, pour ensuite se voir dominé par un dispositif technique autonomisé sur lequel il n'a plus aucune prise. La puissance humaine aliénée sous la forme de la raison instrumentale se matérialise dans la machine qui consiste en l'incarnation technique du capital.

\section{La critique du machinisme}

Dans la première section de cet article, nous avons soutenu que la critique marxienne de la spécificité historique de la domination technique dans le capitalisme se fonde sur son ontologie de l'autoproduction. Cette critique de la technique dans le capitalisme tire une partie de ses influences dans la philosophie d'Aristote portant sur l'inversion du rapport entre idéel et réel chez Platon. Dans cette section, nous verrons que la critique du fétichisme de la marchandise est reprise par Marx pour expliquer la dimension fétichiste du développement technique au sein du capitalisme. C'est cette même inversion que Marx observe lorsqu'il associe le capital à la catégorie de l'esprit au sens hégélien34. Or, comme le note Moishe Postone, pour Marx, le concept hégélien de totalité ne s'applique qu'à la société capitaliste. Seule la société capitaliste possède un principe d'unification totalisant qui s'exprime sous la forme d'une rationalité instrumentale qui s'impose à l'ensemble des sphères de l'activité sociale35. Il est donc malvenu, comme l'ont fait certains commentateurs de Marx, de voir dans le capital la réalisation d'un principe transhistorique qui trouverait son fondement dans l'Antiquité grecque36. Au contraire, la conception de l'histoire comme processus téléologique,

34 Il n'en demeure pas moins que le capital consiste en un mauvais infini en termes hégéliens.

35 Comme nous le verrons dans la section suivante, en dépit de ses grandes qualités, le problème de la relecture de Postone est qu'en écartant de son analyse les écrits du jeune Marx portant sur l'aliénation et l'ontologie sous prétexte qu'ils consisteraient en une conception naturaliste et transhistorique, il est contraint d'adhérer à une lecture progressiste du développement technologique telle qu'on la retrouve dans les Grundrisse. Puisqu'aucune extériorité n'existerait face au capital, Postone n'est pas ainsi en mesure de trouver de fondements normatifs à l'intérieur des sociétés telles qu'elles se sont constituées historiquement permettant de contraindre le développement illimité du dispositif techno-capitaliste.

${ }^{36}$ En reprenant la téléologie de l'histoire de Hegel, Jean Vioulac - à l'instar des analyses d'Alfred SohnRethel suivant lesquelles les catégories universelles et abstraites de la philosophie moderne trouvent leur origine dans la pratique de l'échange marchand de l'Antiquité - considère la domination contemporaine du capital comme la pleine réalisation de la métaphysique platonicienne. Or, l'anthropologie économique nous rappelle que l'échange marchand est dans l'Antiquité une pratique marginale qui ne s'impose pas comme une médiation structurante de la totalité sociale. Ce type d'approche se trouve donc à transhistoriciser des catégories qui appartiennent à une forme historique spécifique, la modernité capitaliste. Voir Jean Vioulac, La logique totalitaire, op. cit. L'analyse transhistorique d'Adorno et Horkeimer, qui s'appuient également sur Sohn-Rethel, dans La Dialectique de la raison (Paris, Gallimard, 1974) mène à la même impasse philosophique. 
mue par un esprit prenant la forme d'un sujet automate, ne s'applique qu'à la société capitaliste. La ressaisie par Marx de la phénoménologie de Hegel dans Le Capital cherche à expliquer les transformations historiques concrètes au sein du mode de production capitaliste qui vont mener à la pleine réalisation du concept de capital compris au sens de l'esprit hégélien.

Marx introduit en effet dans une perspective matérialiste l'idée d'engendrement de soi par soi-même du capital. Pour s'auto-engendrer, le capital doit toutefois trouver devant lui des formes sociales qui ne sont pas posées par lui-même. En se les appropriant, le capital les transformera pour en faire ses propres présuppositions 37. C'est ce qui explique son analyse aux chapitres XI à XIII du Capital, des transformations au sein des modes d'organisation du capitalisme qui vont mener à la pleine réalisation de la catégorie du capital qu'il définit comme « valeur qui s'autovalorise ». En partant de la coopération simple, en passant par la manufacture jusqu'à la grande industrie, une dialectique de l'histoire de l'aliénation de la connaissance humaine et sa matérialisation dans la technologie se déploie. Celle-ci culmine avec la domination du machinisme.

Dans la coopération simple, Marx analyse le premier moment de la soumission du travail au capital qu'il nomme soumission formelle, qui renvoie à la survaleur accumulée par le capital au moyen de l'extension de la journée de travail. Au sein de ce premier moment, les conditions sociales de possibilité du déploiement de la logique $\mathrm{du}$ capital sont mises en place moyennant l'appropriation de la force productive collective découlant de la coopération d'un grand nombre de travailleurs réunis dans un même atelier. C'est à ce stade que se met en place le despotisme d'usine qui repose sur la première forme de machine, la machine humaine qui prend la forme du travailleur collectif38. La catégorie du capital comme sujet automate n'est pas encore pleinement réalisée puisque c'est uniquement à partir de l'exploitation du travail concret que le capital est en mesure d'augmenter la productivité. L'activité du travailleur demeure sensiblement la même que celle qui était effectuée à l'époque artisanale. Si la coopération du procès de travail existe depuis l'Antiquité selon Marx, la spécificité de la forme capitaliste de coopération repose sur l'existence « d'un travailleur salarié libre qui vend sa force de travail au capital $39 »$ ne produisant plus des biens en vue de leur valeur d'usage, mais plutôt de leur valeur d'échange.

Le stade suivant, celui de la manufacture, qu'on peut qualifier de forme de coopération spécifiquement capitaliste, n'entraîne pas une transformation dans le domaine

37 À ce sujet, voir Jacques D’Hondt, « Marx et la phénoménologie », Revue de Métaphysique et de morale, vol. $3, n^{\circ} 55,2017$, p. 289-311.

38 C'est à ce même phénomène que renvoie Lewis Mumford dans la mégamachine, c'est-à-dire les machines qui utilisent les humains comme composantes. Lewis Mumford, Le Mythe de la machine, 2 vol., (1967-1970), Paris, Fayard, 1974.

39 Karl Marx, Le Capital, livre 1, op. cit., p. 376. 
des techniques de production puisque le travailleur continue de maîtriser ses instruments de travail. Néanmoins, elle crée les conditions sociales de possibilité à l'introduction de la machine principalement au moyen de la décomposition du procès de travail général en diverses tâches spécialisées. S'opère alors une dépossession de "l'ensemble de connaissance, de clairvoyance et de volonté40 », qui était le propre de l'artisan, au profit du capital. Bref, c'est de l'abrutissement généralisé des travailleurs que se nourrit la puissance du capital :

Ce que les travailleurs partiels perdent se concentre face à eux, dans le capital. L'un des produits de la division manufacturière du travail est de leur opposer les potentialités spirituelles du procès matériel de production comme une propriété d'autrui et un pouvoir qui les domine. Ce processus de scission commence dans la coopération simple, là où le capitaliste représente face aux travailleurs singuliers l'unité et la volonté du corps du travail social. Il se développe dans la manufacture qui mutile l'ouvrier en faisant un travailleur partiel. Il s'achève dans la grande industrie qui sépare la science, en tant que potentialité productive autonome, du travail, et la met de force au service du capital 41 .

Selon Marx, c'est avec la grande industrie que se met en place le machinisme qui correspond au «mode de production spécifiquement capitaliste 42 », puisqu'il permet d'accumuler de la survaleur relative au moyen de l'augmentation de la productivité et de l'intensification du travail. Dans le machinisme, ce n'est plus l'activité concrète du travailleur qui est la source principale de la création de la richesse, même si le travail demeure au fondement de l'accumulation de la survaleur. C'est plutôt la capacité organisationnelle de la grande entreprise - qui repose sur un savoir technique permettant le contrôle managérial du procès de travail - qui devient le facteur prédominant d'augmentation de la productivité. À ce stade, le travailleur devient un simple appendice de la machine :

La machine, qui est la base de la révolution industrielle, remplace l'ouvrier manipulant son outil singulier, par un mécanisme qui opère en une fois avec quantité de ces outils ou d'outils de même espèce, et qui est mû par une seule force d'actionnement, qu'elle qu'en soit la forme 43 .

L'accélération du cycle de rotation du capital rendue possible par le développement technologique est nécessaire afin, d'une part, d'obtenir des gains de productivité et d'accumuler de la survaleur relative et, d'autre part, afin de raccourcir le temps de

40 Ibid., p. 406.

${ }^{41}$ Ibid., p. 406-407.

42 Ibid., p. 571.

43 Ibid., p. 412. 
circulation, lequel ne produit pas de valeur44. C'est au moment où le procès de valorisation repose sur la survaleur relative que la catégorie du capital prend tout son sens puisque le capital devient le véritable sujet (non humain) d'une dynamique hors de tout contrôle social.

La valeur passe constamment d'une forme dans l'autre, sans se perdre elle-même dans ce mouvement, et elle se transforme en un sujet automate [...] la valeur devient ici le sujet d'un procès dans lequel, à travers le changement constant des formes-argent et marchandise, elle modifie sa grandeur elle-même, se détache en tant que survaleur d'elle-même en tant que valeur initiale, se valorise elle-même45.

Le sujet automate se matérialise dans le système des machines, selon Marx, qui affirme dans une veine aristotélicienne 46 que :

[t]out le système de machinerie, qu'il soit fondé sur la simple coopération de machines de travail analogues, comme dans le tissage, ou sur une combinaison de machines différenciées, constitue en soi un grand automate dès qu'il est mis en mouvement par un premier moteur qui se meut lui-même47.

C'est au stade du machinisme que le fétichisme de la marchandise devient fétichisme de la technique. Lorsque les machines produisent des machines, s'enclenche une autonomisation de l'appareil technique qui prend la forme d'un système autoreproducteur au sein duquel s'opère un renversement de la pratique sociale. La machine est la matérialisation du sujet au sens hégélien qui aspire la force du travailleur réifiée sous la forme d'une ressource humaine. La classe capitaliste ne contrôle plus l'appareil

\footnotetext{
44 Cette distinction entre production et circulation tend à s'estomper dans le capitalisme avancé, et plus précisément à l'ère de la financiarisation. À ce stade, le capital devient réellement unité de la production et de la circulation, ce qui fait que le moment de la circulation participe pleinement à la production de survaleur. À ce sujet voir Paul Baran et Paul Sweezy, «Some theoretical implication », Monthly Review, vol. 64, n 3, 2012 ; en ligne : https://monthlyreview.org/2012/07/01/some-theoretical-implications/; et Edward LiPuma et Benjamin Lee, "Financial derivatives and the rise of circulation », Economy and Society, vol. 34, n 3, 2005, p. 404-427.

45 Karl Marx, Le Capital, livre 1, op. cit., p. 173-174.

46 Plus loin il dira également : « D'où ce phénomène remarquable dans l'histoire de l'industrie moderne : c'est la machine qui fiche en l'air toutes les limites morales et naturelles de la journée de travail. D'où ce paradoxe économique, que le plus puissant moyen de réduction du travail devienne le moyen le plus infaillible pour transformer le temps de vie de l'ouvrier et de sa famille en temps de travail disponible pour la valorisation du capital. "Si" rêvait Aristote, le plus grand penseur de l'Antiquité, "si chaque outil pouvait, sur ordre ou d'instinct, exécuter le travail qui lui échoit, comme les chefs-d'œuvre de Dédale qui se mouvait d'eux-mêmes, ou comme les trépieds d'Héphaïstos qui se mettaient spontanément à leur travail sacré ; si donc les navettes des tisserands se mettaient d'elles-mêmes à tisser, le contremaître n'aurait pas besoin d'aide, ni le maître d'esclaves" » (ibid., p. 458).

47 Ibid., p. 427.
} 
technologique de production; elle ne se compose que de simples fonctionnaires au service de la puissance autonomisée du capital. La dialectique hégélienne entre le maître et l'esclave n'oppose pas tant la classe capitaliste au prolétariat que l'ensemble de l'humanité qui fait face à des structures abstraites qu'elle a constituées, mais sur lesquelles elle n'a pas de prise :

La dextérité et la minutie du travailleur sur la machine vidé de sa substance en tant qu'individu, disparaissent tel un minuscule accessoire devant la science, devant les énormes forces naturelles et le travail social de masse, dont le système des machines est l'incarnation et qui fondent avec lui la puissance du « maître »48.

Selon la lecture de Marx, il est malavisé de postuler une quelconque neutralité de la technique. Elle est plutôt la matérialisation d'une forme de connaissance aliénée qui enclenche une dynamique de croissance illimitée et hors du contrôle humain. Cela signifie qu'à ce stade, la seule réappropriation des moyens de production par les travailleurs ne suffit pas pour opérer un dépassement de la forme aliénée de croissance induite par le capitalisme.

\section{La limite du capital et celles du « marxisme progressiste »}

Le chapitre sur le machinisme nous mène à poser la question fondamentale des limites du capital par-delà l'analyse du marxisme traditionnel qui soutient que la contradiction fondamentale du capitalisme repose sur l'insuffisance du marché à réguler le développement économique de manière rationnelle et équitable (la contradiction entre les rapports de production et les forces productives). C'est pourtant sur ce postulat progressiste que se sont édifiées la théorie du capitalisme cognitif 49 et les thèses accélérationnistes, qui - à partir d'une lecture du passage des Grundrisse communément appelé « Fragment sur les machines »-soutiennent qu'une modification radicale du capitalisme est survenue lors du passage du mode de régulation fordiste vers le postfordisme. Le capitalisme cognitif se caractériserait désormais par l'informatisation de la production qui viendrait remplacer le travail industriel par une nouvelle forme de travail, nommé travail immatériel ou cognitif. Selon Carlo Vercellone, le terme cognitif cherche à rendre compte de la nature nouvelle du travail au sein de laquelle « la valeur réside désormais dans les savoirs mobilisés par le travail vivant et non dans les ressources et le travail matériel5o ». Les « cognitivistes » appuient leur thèse sur le constat suivant lequel «l'activité de travail d'une partie croissante de la population

48 Ibid., p. 475.

49 Parmi les théoriciens du capitalisme cognitif, on retrouve, entre autres, Antonio Negri, Michael Hardt, Carlos Vercellone et Yann Moulier-Boutang.

$5^{\circ}$ Carlos Vercellone, «Travail, information et connaissance dans le nouveau capitalisme. La thèse du capitalisme cognitif », dans Critiques de la société de l'information, dir. Éric George et Fabien Granjon, Paris, L’Harmattan, 2008, p. 234. 
consiste toujours davantage à traiter de l'information, à produire des connaissances et à s'engager dans des relations de services fondées sur l'échange de savoirs, la communication et la production de l'homme par l'homme ${ }^{1}{ }^{\prime} \gg$. Dans ce contexte, soutiennentils, la théorie marxienne de la valeur-travail serait désuète puisque le capital ne serait pas en mesure de calculer le travail immatériel comme c'était possible pour le travail manuel effectué en usine. Comme le travail immatériel ne se bornerait pas aux frontières de l'usine, c'est l'ensemble de la société qui deviendrait une usine et qui produirait de la valeur. La théorie de la valeur-travail laisserait donc sa place à la théorie de la valeur-savoir. Les théoriciens du capitalisme cognitif fondent leur théorie de la valeur-savoir sur ce passage des Grundrisse :

à mesure que la grande industrie se développe, la création de richesses dépend de moins en moins du temps de travail utilisé, et de plus en plus de la puissance des agents mécaniques qui sont mis en mouvement pendant la durée de travail [...]. Elle dépend bien plutôt du niveau général de la science et du progrès de la technologie, ou de l'application de cette science à cette production [...]. Dès que le travail, sous sa forme immédiate, a cessé d'être la source principale de la richesse, le temps de travail cesse et doit cesser d'être sa mesure, et la valeur d'échange cesse donc aussi d'être la mesure de la valeur d'usage ${ }^{2}$.

Toutefois, ce passage des Grundrisse a été compris dans une perspective ricardienne par les théoriciens du capitalisme cognitif. Selon Ricardo, c'est le travail concret qui vient directement constituer la valeur d'une marchandise. Pour Marx, au contraire, c'est le travail abstrait en tant qu'il est représenté par une norme correspondant à la moyenne de temps pris pour produire une marchandise (le temps de travail moyen) qui fonde la valeur. Les théoriciens du capitalisme cognitif confondent ainsi les concepts de travail immatériel et de travail abstrait, de même que ceux de richesse et de valeur. Il est essentiel de distinguer ces catégories, sans quoi cela mène à des propositions politiques contestables d'un point de vue écologique puisqu'il s'agirait au final de développer de manière illimitée des forces productives pour sortir automatiquement du capitalisme. Or, par-delà les contradictions objectives du capital, le développement illimité du capitalisme possède avant tout une limite objective, celle de la nature et de l'être humain :

la production capitaliste ne développe la technique et la combinaison du procès de production sociale qu'en ruinant dans le même temps les sources vives de toute richesse : la terre et le travailleur53.

\footnotetext{
${ }^{51}$ Ibid., p. 235.

$5^{2}$ Karl Marx, Fondements de la critique de l'économie politique, op. cit., p. 221-222.

53 Karl Marx, Le Capital, livre 1, op. cit., p. 567.
} 
Contrairement aux cognitivistes, Moishe Postone soutient qu'au sein du capitalisme avancé, ce n'est plus le travail concret du travailleur qui est la principale source de la productivité, c'est plutôt le temps historique objectivé, lequel correspond au patrimoine intellectuel, culturel et scientifique de l'humanité, ce que Marx nomme dans les Grundrisse le general intellect54. Si en effet, avec le développement technologique ce n'est plus l'activité immédiatement effectuée par les travailleurs qui est au fondement de la production de la richesse, la médiation des rapports sociaux au moyen du travail demeure de manière purement archaïque la source principale de la valeur. Selon Postone, il ne s'agit pas pour Marx d'affirmer positivement ce qui existe sous une forme aliénée via le développement exponentiel des forces productives, mais plutôt de voir qu'il s'agit d'une limite inhérente au capital qui permet d'envisager une contradiction entre ce qui est et ce qui pourrait être si les rapports sociaux cessaient d'être médiatisés par la forme valeur et le travail abstrait. Marx soulève cette contradiction dans un passage des Grundrisse :

Le capital est une contradiction en procès : d'une part, il pousse à la réduction du temps de travail à un minimum et, d'autre part, il pose le temps de travail comme la seule source et la seule mesure de la richesse. Il diminue donc le temps de travail sous sa forme nécessaire pour l'accroître sous sa forme de surtravail. Dans une proportion croissante, il pose donc le surtravail comme la condition (question de vie ou de mort) du travail nécessaire [...]. Le développement du capital fixe indique le degré où la science sociale en général, le savoir sont devenus une force productive immédiate et, par conséquent, jusqu'à quel point les conditions du procès vital de la société sont soumises au contrôle de l'intelligence générale et portent sa marque; jusqu'à quel point les forces productives locales ne sont pas seulement produites sous la forme du savoir, mais encore comme organes immédiats de la praxis sociale, du procès vital réel55.

Or, les théoriciens du capitalisme cognitif, qui réintroduisent la notion de general intellect dans leur analyse du rôle de la technologie dans les transformations du capitalisme, interprètent cette notion de manière non dialectique. Il ne s'agirait plus, selon eux, d'instituer un au-delà à la médiation des rapports sociaux par le travail, mais plutôt d'affirmer positivement ce qui existe sous une forme aliénée. En clair, il suffirait simplement de se débarrasser de la médiation parasitaire de la propriété privée des moyens de production et du marché, pour réaliser immédiatement le potentiel productif de la multitude. Cette «libération» du travail permettrait de concrétiser l'idéal communiste qui se caractériserait justement par l'absence de médiations sociales.

54 Moishe Postone, Temps, travail et domination sociale, Paris, Mille et une nuits, 2009.

55 Karl Marx, Fondements de la critique de l'économie politique, op. cit., p. 222. 
On peut percevoir ici une limite historique des analyses marxistes qui s'appuient sur les Grundrisse, comme celle proposée par les théoriciens du capitalisme cognitif et les accélérationnistes, mais aussi par les théoriciens de la critique de la valeur comme Moishe Postone. Toutes ces approches, en dépit de leurs importantes divergences, reposent sur des postulats progressistes qui peinent à penser les voies de dépassement vers une forme de société où la croissance du capital et de l'appareil technoscientifique ne mènerait pas à la destruction de l'humain et de la nature56. Le problème de ce type d'analyses repose sur l'absence d'une critique dialectique des modes de connaissance tels que constitués dans le capitalisme. Une théorie critique du rôle de la technique dans le capitalisme se doit de montrer qu'au niveau de la société comprise comme totalité, le capital génère un procès d'aliénation des modes de connaissance et d'expériences sociales et symboliques en général, aliénation tributaire d'une " dialectique des Lumières », pour reprendre les termes de Max Horkheimer et de Theodor Adorno57.

En effet, au moment où Marx écrit, ni la culture, ni la science, ne sont encore soumises à un dispositif industriel de production massifié avec ses indicateurs de performance et sa division du travail, c'est-à-dire qu'il ne s'agit pas encore d'une recherche technoscientifique. Pour Marx, les découvertes scientifiques se situent en extériorité au capital, elles lui offrent un service gratuit: «Une fois découvertes, les lois physiques qui régissent la déviation de l'aiguille aimantée dans le champ d'action d'un courant électrique, ou la production du magnétisme dans le fer autour duquel circule un courant électrique ne coûtent pas un liard $58 \gg$. Or, les transformations institutionnelles marquant le passage vers le capitalisme avancé se caractérisent justement par l'intégration de plus en plus marquée de la culture en général, et de la production de la connaissance en particulier, par la logique de la valorisation du capital. En termes hégéliens, on peut dire que si le capital a trouvé dans la science un matériau sous forme d'une présupposition permettant son expansion, cette présupposition n'était pas posée par lui. Le capital ne tolère pas les présuppositions qui ne sont pas posées par lui, pour les rendre conformes à son concept, il doit se les approprier pour en faire sa propre présupposition59. On voit donc mal comment le savoir accumulé par l'humanité, qui est désormais approprié par l'appareil technoscientifique, pourrait nous permettre, comme le soutient Postone, d'envisager un dépassement du capitalisme.

Cette dialectique négative de la raison est le résultat de l'émancipation par la technique spécifiquement capitaliste. Comme le rappelle Jean Vioulac, le terme

\footnotetext{
56 Pour une critique des thèses de Postone d'un point de vue écologique, voir Fabrice Flipo, « Moishe Postone, une lecture de Marx (enfin) débarrassée du productivisme ? », Mouvements, nº 60, 2009, p. 146151.

57 Theodor Adorno et Max Horkheimer, La dialectique de la raison, op. cit. ; le titre original, mal traduit en français, est Dialektik der Aufklärung.

$5^{8}$ Karl Marx, Le Capital, livre 1, op. cit., p. 434.

59 Jacques D’Hondt, « Marx et la phénoménologie », op. cit., p. 289-311.
} 
émancipation provient du latin ex manu capere, qui veut dire se laisser prendre des mains quelque chose, s'en dé-saisir et ainsi lâcher prise ${ }^{60}$. Cette émancipation a dépossédé l'humain du maniement de ses outils et de son entendement, donc de sa raison, qui est désormais automatisée. On passe dès lors de l'aliénation du travail manuel en miettes à celle du « travail intellectuel en miettes ${ }^{61} »$. En témoigne le développement contemporain des mécanismes automatisés et autorégulés de prise de décision opérés par des algorithmes auto-apprenants dans l'ensemble des secteurs de la société : la finance, le marketing, la santé, l'éducation, etc. En ce sens le déploiement de l'intelligence artificielle annonce un gigantesque processus de prolétarisation, c'est-à-dire la dépossession, l'extériorisation et la captation par la machine de l'ensemble des savoirfaire, des savoir-vivre et des savoirs théoriques au fondement de l'essence symbolique de l'être humain ${ }^{62}$. Face à cette aliénation généralisée et aux conséquences écocides à laquelle elle conduit, il apparaît plutôt irresponsable de miser sur la croissance illimitée des forces productives ou encore sur le développement du general intellect qui a été accaparé par la technoscience pour fonder une société post-capitaliste.

\section{Penser les limites face à l'hubris du capital technoscientifique}

La critique du développement technologique chez Marx doit être située dans le contexte de la mise en place de la révolution industrielle au XIX ${ }^{\mathrm{e}}$ siècle. Comme nous l'avons montré, contrairement à ce que soutient le marxisme traditionnel, Marx ne peut être considéré comme un penseur du déterminisme technologique qui envisage la croissance illimitée de l'appareil productif comme une voie de passage automatique vers une société post-capitaliste. Il reste qu'en dépit de la dénonciation du caractère déshumanisant de la technique moderne alimentée par le principe machiniste, il voit dans le développement capitaliste un progrès par rapport aux formes sociales antérieures et ne croit pas possible ni envisageable un retour vers un mode de production artisanal. Il ne nous laisse malheureusement que très peu d'indices sur ce que pourrait être un autre rapport à la technique dans une société post-capitaliste ${ }^{63}$. En dépit de ses limites, la critique de la technique chez Marx s'avère malgré tout d'une étonnante actualité surtout si on la considère à partir de son ontologie de l'autoproduction. La prise en considération de l'influence aristotélicienne dans la pensée de Marx peut nous aider réfléchir à la nécessité de poser des limites face à l'hubris de la logique de croissance capitaliste, et donc d'envisager quelles formes pourraient prendre un socialisme

60 Jean Vioulac, L'époque de la technique, op. cit., p. 302.

${ }^{61}$ Michel Freitag, Le naufrage de l'université et autres essais d'épistémologie politique, Québec, Nota bene, 1998, p. 80.

62 Bernard Stiegler, États de choc. Bêtise et savoir au XXI siècle, Paris, Mille et une nuits, 2012.

63 C'est à cette question que s'est affairée Marcuse de façon plus ou moins convaincante dans sa critique de la société industrielle. À ce sujet, voir Andrew Feenberg, Philosophie de la praxis. Marx, Lukács et l'École de Francfort, Montréal, Lux, 2016. 
sans croissance ${ }^{64}$. La question des limites peut aussi nous éclairer sur certains points aveugles qui hantent le marxisme et la pensée de Marx elle-même. En effet, Marx demeure un penseur de son temps, obsédé par l'idée proprement moderne d'émancipation, mais qui peine à penser positivement la chose publique ${ }^{65}$, ou encore les médiations permettant la reproduction sociale ${ }^{66}$. Les développements contemporains du capitalisme et de la technologie - qu'on ne peut désormais plus opposer - nous invitent donc à repenser autrement l'émancipation ; c'est-à-dire comme une capacité collective d'établir des limites face à l'hubris du capital.

Les élans progressistes de Marx dans les Grundrisse sont d'ailleurs tempérés dans Le Capital où, par-delà l'eschatologie révolutionnaire, il évoque la nécessité d'imposer des limites temporelles à l'expansion infinie du capital. Cette stratégie « réformiste » peut être interprétée comme la mise en place des conditions de possibilité permettant une ressaisie réflexive de la dynamique aliénée du capital :

Après moi le déluge ! Telle est la devise de tout capitaliste et de toute nation capitaliste. Le capital n'a donc aucun scrupule s'agissant de la santé et de l'espérance de vie de l'ouvrier. S'il n'y est pas contraint par la société67.

C'est peut-être en ce sens que Marx caractérisait, à la fin du livre 3 du Capital, la société post-capitaliste par sa capacité à s'autolimiter; l'autoproduction ne pouvant être possible que lorsqu'elle s'effectue dans le respect des limites de la nature humaine et de l'environnement :

À la vérité, le règne de la liberté commence seulement à partir du moment où cesse le travail dicté par la nécessité et les fins extérieures; il se situe donc, par sa nature même, au-delà de la sphère de la production matérielle proprement dite [...]. Dans ce domaine, la liberté ne peut consister qu'en ceci : les producteurs associés - l'homme socialisé - règlent de manière rationnelle leurs échanges organiques avec la nature et les soumettent à leur contrôle commun au lieu d'être dominés par la puissance aveugle de ces échanges; et ils accomplissent en dépensant le moins d'énergie

\footnotetext{
64 À ce sujet, voir Éric Pineault, « From provocation to challenge : Degrowth, capitalism and the prospect of "socialism without growth", a commentary on Giorgio Kallis », Capitalism, nature, socialism, vol. 30, $\mathrm{n}^{\mathrm{o}} 2$ 2, 2019, p. 251-266.

65 Domenico Losurdo, «Avec Gramsci, par-delà Marx et par-delà Gramsci », Nouvelles FondationS, vol. $3, n^{\text {os }} 7-8,2007$, p. 210-218.

66 Nancy Fraser soutient que la pensée de Marx sur l'émancipation contient des points aveugles qui doivent être complétés par l'analyse de Karl Polanyi portant sur les mécanismes d'autoprotection des sociétés face à l'utopie d'un système économique autorégulé. Nancy Fraser, « Why two Karls are better than one : Integrating Polanyi and Marx in a critical theory of the current crisis », Working Paper der DFG-Kollegforscher_innengruppe Postwachstumsgesellschaften, $\mathrm{n}^{0}$ 1, 2017.

67 Karl Marx, Le Capital, livre 1, op. cit., p. 301 ; nous soulignons.
} 
possible, dans les conditions les plus dignes, les plus conformes à leur nature humaine. Mais l'empire de la nécessité n'en subsiste pas moins. C'est au-delà que commence l'épanouissement de la puissance humaine qui est sa propre fin, le véritable règne de la liberté qui, cependant, ne peut fleurir qu'en se fondant sur ce règne de la nécessité. La réduction de la journée de travail est la condition fondamentale de cette libération 68 .

Si ce passage laisse présager l'utopie d'une société des loisirs, celle-ci ne réfère certainement pas à la société de consommation de masse abrutissante. Comme le rappelle Pierre Rodrigo, il ne s'agit pas «d'améliorer l'exploitation capitaliste du travail vivant en y ménageant, grâce au machinisme, du temps de loisirs ! 69 », mais plutôt de rappeler que le loisir chez les Grecs se nomme skholè, ce qui signifie le temps consacré aux études, à l'élévation de l'âme et de l'esprit grâce à la culture. C'est dans le ralentissement du dispositif de production, donc en imposant politiquement des limites, qu'il est possible de ressaisir réflexivement l'ensemble des médiations sociales aliénées sous le capitalisme. Or ces limites instituées politiquement ne peuvent s'appuyer que sur des normes culturelles et symboliques qui se sont constituées historiquement par les sociétés humaines en extériorité au capital. On voit mal comment elles pourraient surgir du mode de vie consumériste qui a été sciemment construit par le capital.

En effet, si sous le règne de la nécessité notre rapport à la technique, à la science et à la connaissance de l'humain en général ne peut être que réifié, ce n'est que sous le règne de la liberté, dit Jacques D'Hondt, qu'il est possible d'entrevoir un autre rapport à la connaissance de l'homme qui ne sera peut-être même plus scientifique (au sens où l'entend la rationalité quantifiante moderne70). Selon Constazo Preve, l'intention normative de Marx est de rétablir le primat de la "science philosophique » sur la « science » telle qu' on la conçoit dans le monde moderne, puisque la science, contrairement à la philosophie, est incapable de poser ses questions normativement :

La science de Marx est par conséquent une science philosophique, dans le sens précis qui est donné à ce terme dans la Phénoménologie de l'Esprit, et dans la Science de la Logique de Hegel. [...] Que signifie la science philosophique? Cela veut dire: savoir absolu qui allie l'élément de la connaissance, qui est conceptualisation d'un objet de connaissance, avec l'élément de son évaluation éthique et morale. Pour ce qui est de Marx, il s'agit du mode de production capitaliste entendu selon son articulation interne, sa

\footnotetext{
68 Karl Marx, Le Capital, livre 3 : Le procès d'ensemble de la production capitaliste, dans Cuvres, t. 2, Paris, Gallimard, coll. « La Pléiade », 1968, p. 1487-1488.

69 Pierre Rodrigo, « Marx et la technique », op. cit., p. 50.

70 Jacques D’Hondt, «La connaissance de l'humain selon Marx », dans Marx et les sciences humaines. Actes du colloque de Neuchâtel, dir. Gerhard Seel, Lausanne, L'Âge d'homme, 1987, p. 54-74.
} 
genèse historique et le processus concret global de sa reproduction et de son développement, aussi bien que selon l'aliénation, l'exploitation et la polarité géographique mondiale entre riches et pauvres, etc. Telle est la science philosophique71.

La réappropriation du temps libre est donc la condition transcendantale de possibilité afin que la philosophie remplace l'économie politique comme forme de connaissance structurant normativement la pratique sociale, et que la cité cesse d'être gouvernée par la mégamachine ${ }^{72}$. Ce qui revient à dire qu'il est possible, souhaitable et nécessaire de s'opposer au " progrès » contrairement aux chantres de l'innovation pour qui « on ne peut pas arrêter le progrès 73 ». En ce sens, la décélération 74 est la condition de possibilité à la question philosophique et politique fondamentale posée par Aristote dans l'Antiquité : qu'est-ce que la vie bonne?

\footnotetext{
${ }^{71}$ Constanzo Preve, Une nouvelle histoire alternative de la philosophie. Le chemin ontologico-social de la philosophie, Paris, Perspectives libres, 2017, p. 389.

72 Lewis Mumford, Le Mythe de la machine, op. cit.

73 David Noble, Progress without people. New technology, unemployment and the message of resistance, Toronto, Between the Lines, 1995.

74 À ce sujet voir Hartmut Rosa, Résonance. Une sociologie de la relation au monde, Paris, La Découverte, 2018.
} 